\title{
LOCI OF RATIONAL CURVES OF SMALL DEGREE ON THE MODULI SPACE OF VECTOR BUNDLES
}

\author{
INSONG CHOE
}

\begin{abstract}
For a smooth algebraic curve $C$ of genus $g \geq 4$, let $S U_{C}(r, d)$ be the moduli space of semistable bundles of rank $r \geq 2$ over $C$ with fixed determinant of degree $d$. When $(r, d)=1$, it is known that $S U_{C}(r, d)$ is a smooth Fano variety of Picard number 1, whose rational curves passing through a general point have degree $\geq r$ with respect to the ample generator of $\operatorname{Pic}\left(S U_{C}(r, d)\right)$. In this paper, we study the locus swept out by the rational curves on $S U_{C}(r, d)$ of degree $<r$. As a by-product, we present another proof of Torelli theorem on $S U_{C}(r, d)$.
\end{abstract}

\section{Introduction}

Let $C$ be a smooth algebraic curve over $\mathbb{C}$ of genus $g \geq 4$. Let $\mathcal{M}:=$ $S U_{C}(r, d)$ be the moduli space of semistable bundles of rank $r \geq 2$ over $C$ with fixed determinant of degree $d$. Throughout this paper, we assume $(r, d)=1$. In this case, it is known that $\mathcal{M}$ is a smooth Fano variety of Picard number 1. Hence it is an important project to study the rational curves on $\mathcal{M}$ using the modular properties.

In general, for a Fano manifold $N$ of Picard number 1, the index of $N$ is defined by the number $i$ such that $-K_{N} \cong \mathcal{O}_{N}(i)$, where $\mathcal{O}_{N}(1)$ is the ample generator of $\operatorname{Pic}(N)$. In analogy with the case of projective hypersurfaces, a rational curve $l \subset N$ is called a line on $N$ if the index of $N$ equals $-K_{N} \cdot l$. Also we say that $l$ has degree $k$ if $-K_{N} \cdot l$ equals $k$ times the index of $N$. It is an open question if every Fano manifold of Picard number 1 has a line ([3], p. 248).

As a Fano manifold, $\mathcal{M}$ has index 2 ([1]). Ramanan [7] found a family of lines on $\mathcal{M}$, but they sweep out a sublocus of $\mathcal{M}$ of large codimension. It has also been observed that $\mathcal{M}$ is covered by rational curves of degree $r$, which are called Hecke curves ([6], Corollary 5.16). Hwang ([2], Question 1) asked if the Hecke

Received August 5, 2009.

2010 Mathematics Subject Classification. 14C34, $14 \mathrm{H} 60$.

Key words and phrases. rational curves, moduli of vector bundles over a curve, scroll, Torelli theorem.

This work was supported by the National Research Foundation of Korea(NRF) grant funded by the Korea government(MEST) (No. 2010-0001194). 
curves are the rational curves of minimal degree passing through a general point of $\mathcal{M}$. This was recently answered affirmatively by Sun ([9], Theorem 1). As a corollary, he gave another proof of nonabelian Torelli theorem. He also proved that there are no lines on $\mathcal{M}$ different from those found by Ramanan ([9], Theorem 2).

The aim of this paper is to get more detailed information on the rational curves on $\mathcal{M}$ of degree between 1 and $r$. In particular, we show that the locus inside $\mathcal{M}$ swept out by the rational curves of degree $<r$, consists of $r-1$ irreducible components, each of which comes from certain extensions of fixed type. We also study the intersections of these components. As a by-product, we get another way to recover the curve $C$ from the moduli space $\mathcal{M}=S U_{C}(r, d)$.

This paper is organized as follows. In $\S 2$, we study certain extension spaces parameterizing the bundles which are not $(1,1)$-stable. It will turn out that these spaces provide the locus of rational curves of small degree.

In $\S 3$, we study the intersections of the images of the extension spaces inside $\mathcal{M}$. In most cases, their intersection is empty, but there are special pairs whose intersection property can be described in terms of certain scroll over $C$.

In $\S 4$, we first briefly review Sun's results on rational curves on $\mathcal{M}$. Combining with the informations from $\S 2$ and $\S 3$, we prove in Proposition 4.2 that for every $\varepsilon$ with $1 \leq \varepsilon \leq r-1$, the locus in $\mathcal{M}$ swept out by the rational curves of degree $\leq \varepsilon$ consists of $\varepsilon$ irreducible components. Finally in Proposition 4.5, we observe that these results on the loci of the rational curves of small degree provide us another proof of Torelli theorem.

\section{Extension spaces}

From now on, we fix a curve $C$ of genus $g \geq 4$ and simplify our notation so that $U(\rho, \delta)$ denotes the moduli space of semistable bundles over $C$ of rank $\rho$ and degree $\delta$. Let $U^{s}(\rho, \delta)$ be the open subset of $U(\rho, \delta)$ consisting of stable bundles. Also for a line bundle $\Lambda$ on $C$, let $S U(\rho, \Lambda)$ be the moduli space of semistable bundles over $C$ of rank $\rho$ with determinant $\Lambda$.

Definition 2.1. (1) For each $0<\rho^{\prime}<\rho$ and $W \in U^{s}(\rho, \delta)$, define

$$
s_{\rho^{\prime}}(W):=\min \left\{\rho^{\prime} \delta-\rho \operatorname{deg}\left(W^{\prime}\right)\right\},
$$

where the minimum is taken over the subbundles $W^{\prime}$ of $W$ of rank $\rho^{\prime}$.

(2) For any fixed integers $s \geq 0$ and $\rho^{\prime}$, we define

$$
U_{\rho^{\prime}, s}(\rho, \delta):=\left\{W \in U^{s}(\rho, \delta): s_{\rho^{\prime}}(W)>s\right\} .
$$

(3) We say that a bundle $W \in U(\rho, \delta)$ is Lange-stable if $W \in U_{\rho^{\prime}, s}(\rho, \delta)$ where $s=\rho^{\prime}\left(\rho-\rho^{\prime}\right)(g-1)-1$ for every $\rho^{\prime}$.

(4) A bundle $W \in U(\rho, \delta)$ is called $(1,1)$-stable if $W \in U_{\rho^{\prime}, \rho}(\rho, \delta)$ for every $\rho^{\prime}$ (cf. [6], Definition 5.1). 
Lemma 2.2. (1) For each $\rho^{\prime}$, if $s<\rho^{\prime}\left(\rho-\rho^{\prime}\right)(g-1)$, then $U_{\rho^{\prime}, s}(\rho, \delta)$ and $U_{\rho^{\prime}, s}(\rho, \delta) \cap S U(\rho, \Lambda)$ are nonempty open subsets of $U(\rho, \delta)$ and $S U(\rho, \Lambda)$ respectively.

(2) A general bundle $W \in S U(\rho, \Lambda)$ is Lange-stable and $(1,1)$-stable.

Remark 2.3. The following inequalities on the slope $\mu(W):=\operatorname{deg}(W) / \operatorname{rk}(W)$ will be used in the proof below.

(1) For $s=\rho^{\prime}\left(\rho-\rho^{\prime}\right)(g-1)-1$, the inequality defining $U_{\rho^{\prime}, s}(\rho, \delta)$ implies

$$
\mu(W)-\mu\left(W^{\prime}\right) \geq 3\left(\rho-\rho^{\prime}\right) / \rho,
$$

since $g \geq 4$. Also, we note that $2\left(\rho-\rho^{\prime}\right) / \rho \geq 1 / \rho^{\prime}$ for any $1 \leq \rho^{\prime}<\rho$.

(2) The inequality defining $(1,1)$-stability is equivalent to $\mu(W)-\mu\left(W^{\prime}\right)>$ $1 / \rho^{\prime}$.

Proof. (1) By dimension count, one can show that a general bundle $W \in U(\rho, \delta)$ satisfies

$$
s_{\rho^{\prime}}(E) \geq \rho^{\prime}\left(\rho-\rho^{\prime}\right)(g-1),
$$

see [4], p. 448. The statement for $S U(\rho, \Lambda)$ follows from the observation that for every line bundle $L$ of degree 0 , we have $s_{\rho^{\prime}}(W)=s_{\rho^{\prime}}(W \otimes L)$.

(2) The statement on Lange-stability is an immediate consequence of (1). Also by Remark 2.3, Lange-stability implies $(1,1)$-stability.

Now we consider the bundles $V \in \mathcal{M}=S U(r, d)$ which are not $(1,1)$-stable, or equivalently, the bundles which admit a subbundle $V_{1}$ such that

$$
\operatorname{rk}\left(V_{1}\right) d-r \operatorname{deg}\left(V_{1}\right) \leq r .
$$

Here the equality cannot hold since $(r, d)=1$. For each integer $0<\varepsilon<r$, let $r_{1}$ and $d_{1}$ be the integers satisfying $0<r_{1}<r$ and $r_{1} d-r d_{1}=\varepsilon$. Let $r_{2}=r-r_{1}$ and $d_{2}=d-d_{1}$. For $V_{1} \in U\left(r_{1}, d_{1}\right)$ and $V_{2} \in U\left(r_{2}, d_{2}\right)$, consider those bundles $V$ associated with nontrivial extensions

$$
0 \rightarrow V_{1} \rightarrow V \rightarrow V_{2} \rightarrow 0 .
$$

Note the following identities: for $0<r_{1}, r_{2}, \varepsilon<r$,

$$
r_{1} d-r d_{1}=r d_{2}-r_{2} d=r_{1} d_{2}-r_{2} d_{1}=\varepsilon .
$$

We have the following which generalizes and refines [7], Lemma 2.1 and [9], Lemma 3.1.

Lemma 2.4. Suppose that $V_{1}$ and $V_{2}$ in the nontrivial extension (2.1) are Lange-stable. Then for a proper subbundle $V^{\prime}$ of $V$ of rank $r^{\prime}$, the inequality

$$
\mu(V)-\mu\left(V^{\prime}\right)<1 / r^{\prime}
$$

implies that $\operatorname{rk}\left(V^{\prime}\right)=r_{2}, \operatorname{deg}\left(V^{\prime}\right)=d_{2}-1$, and the composition $V^{\prime} \rightarrow V \rightarrow V_{2}$ is a sheaf injection, unless $V^{\prime}$ coincides with the subbundle $V_{1}$. In particular, $V \in U_{r^{\prime}, r}(r, d)$ for each $r^{\prime} \neq r_{1}, r_{2}$. 
Proof. We need to show that

$$
\mu(V)-\mu\left(V^{\prime}\right)>1 / r^{\prime}
$$

for every subbundle $V^{\prime}$ of rank $r^{\prime}$ and degree $d^{\prime}$ if $V^{\prime}$ is neither $V_{1}$ nor a bundle of rank $r_{2}$ with the specified properties. This can be checked case by case. Let $V_{2}^{\prime}$ be the image of the composition $V^{\prime} \rightarrow V \rightarrow V_{2}$ and let $V_{1}^{\prime}$ be the kernel of $V^{\prime} \rightarrow V_{2}^{\prime}$. Let $r_{1}^{\prime}$ and $d_{1}^{\prime}$ (resp. $r_{2}^{\prime}$ and $d_{2}^{\prime}$ ) be the rank an degree of $V_{1}^{\prime}$ (resp. $\left.V_{2}^{\prime}\right)$.

(i) First assume $V_{2}^{\prime}=0$ and $V^{\prime}=V_{1}^{\prime} \neq V_{1}$. Since $V^{\prime}$ is a subbundle of $V$, $r^{\prime}=r_{1}^{\prime}<r_{1}$. Hence by the identity $(2.2)$ and the $(1,1)$-stability of $V_{1}$, we get

$$
\begin{aligned}
\mu(V)-\mu\left(V^{\prime}\right) & =\mu(V)-\mu\left(V_{1}\right)+\mu\left(V_{1}\right)-\mu\left(V^{\prime}\right) \\
& >\frac{\varepsilon}{r r_{1}}+\frac{1}{r_{1}^{\prime}}>\frac{1}{r_{1}^{\prime}}=\frac{1}{r^{\prime}} .
\end{aligned}
$$

(ii) Next assume $V_{1}^{\prime}=0$ and $V^{\prime}=V_{2}^{\prime} \neq V_{2}$. If $r_{2}^{\prime}=r_{2}$, then $d_{2}^{\prime}<d_{2}$ and

$$
\mu(V)-\mu\left(V^{\prime}\right)=\frac{r_{2} d-r d_{2}^{\prime}}{r r_{2}}=\frac{r\left(d_{2}-d_{2}^{\prime}\right)-\varepsilon}{r r_{2}} .
$$

Thus $\mu(V)-\mu\left(V^{\prime}\right)>1 / r_{2}=1 / r^{\prime}$ if and only if $d_{2}-d_{2}^{\prime} \geq 2$.

If $r_{2}^{\prime}<r_{2}$, by the similar argument as (i), we get $\mu(V)-\mu\left(V^{\prime}\right)>1 / r_{2}^{\prime}=1 / r^{\prime}$.

(iii) Now assume $0 \neq V_{1}^{\prime} \neq V_{1}$ and $V_{2}^{\prime}=V_{2}$. By Remark 2.3 (1) and (2.2), we get

$$
\begin{aligned}
\mu\left(V_{1}^{\prime}\right) & =\left(\mu\left(V_{1}^{\prime}\right)-\mu\left(V_{1}\right)\right)+\mu\left(V_{1}\right) \\
& \leq-\frac{3\left(r_{1}-r_{1}^{\prime}\right)}{r_{1}}+\mu(V)-\frac{\varepsilon}{r r_{1}} .
\end{aligned}
$$

Thus,

$$
\begin{aligned}
r^{\prime} \mu\left(V^{\prime}\right) & =\mu\left(V_{1}^{\prime}\right) r_{1}^{\prime}+\mu\left(V_{2}\right) r_{2} \\
& \leq\left(\mu(V)-\frac{3\left(r_{1}-r_{1}^{\prime}\right)}{r_{1}}-\frac{\varepsilon}{r r_{1}}\right) r_{1}^{\prime}+\left(\mu(V)+\frac{\varepsilon}{r r_{2}}\right) r_{2} \\
& =r^{\prime} \mu(V)-\frac{3\left(r_{1}-r_{1}^{\prime}\right)}{r_{1}} \cdot r_{1}^{\prime}-\frac{r_{1}^{\prime} \varepsilon}{r r_{1}}+\frac{\varepsilon}{r} \\
& <r^{\prime} \mu(V)-\frac{2\left(r_{1}-r_{1}^{\prime}\right)}{r_{1}} \cdot r_{1}^{\prime}-\frac{\left(r_{1}-r_{1}^{\prime}\right)}{r_{1}} \cdot r_{1}^{\prime}+\frac{\left(r_{1}-r_{1}^{\prime}\right)}{r_{1}} \\
& \leq r^{\prime} \mu(V)-\frac{2\left(r_{1}-r_{1}^{\prime}\right)}{r_{1}} \cdot r_{1}^{\prime} \\
& \leq r^{\prime} \mu(V)-1 .
\end{aligned}
$$

(iv) The remaining cases $\left(V_{1}^{\prime}=V_{1}, 0 \neq V_{2}^{\prime} \neq V_{2}\right)$ and $\left(0 \neq V_{1}^{\prime} \neq V_{1}, 0 \neq\right.$ $V_{2}^{\prime} \neq V_{2}$ ) can be checked similarly.

For a fixed line bundle $\Lambda \in \operatorname{Pic}^{d}(C)$, consider the subvariety of $U\left(r_{1}, d_{1}\right) \times$ $U\left(r_{2}, d_{2}\right)$ defined by

$$
\mathcal{D}_{\varepsilon}:=\left\{\left(V_{1}, V_{2}\right): \operatorname{det} V_{1} \otimes \operatorname{det} V_{2} \cong \Lambda\right\},
$$


where $r_{1}, d_{1}$ and $r_{2}, d_{2}$ are determined by $\varepsilon$ and the identities (2.2). Let $P\left(V_{1}, V_{2}\right):=\mathbb{P} H^{1}\left(C, V_{2}^{*} \otimes V_{1}\right)$ be the projective space parameterizing the nontrivial extensions up to nonzero scalars.

Lemma 2.5. If $\left(V_{1}, V_{2}\right) \in \mathcal{D}_{\varepsilon}$ is general, then the classifying map

$$
\Phi: P\left(V_{1}, V_{2}\right) \rightarrow S U(r, \Lambda)
$$

is an injective morphism.

Proof. We assume that $V_{1}$ and $V_{2}$ are Lange-stable. By Lemma 2.4, every bundle $V$ associated to a point of $P\left(V_{1}, V_{2}\right)$ is stable. We claim that there exists a unique injection $V_{1} \rightarrow V$ up to homothety, which implies that $\Phi$ is an injective morphism. When $r_{1} \neq r_{2}$, this was already shown in Lemma 2.4.

Now assume $r_{1}=r_{2}$ and suppose that there are two different subbundle maps, say $\alpha, \beta: V_{1} \rightarrow V$, where $\alpha$ induces the original exact sequence

$$
0 \rightarrow V_{1} \stackrel{\alpha}{\rightarrow} V \rightarrow V_{2} \rightarrow 0 \text {. }
$$

Applying Lemma 2.4 for $V^{\prime}=\left(\beta: V_{1} \rightarrow V\right)$, we deduce that $d_{1}=d_{2}-1$ and the composition $V_{1} \stackrel{\beta}{\rightarrow} V \rightarrow V_{2}$ is a sheaf injection. This implies that the induced map $\alpha \oplus \beta: V_{1} \oplus V_{1} \rightarrow V$ is also a sheaf injection, whose quotient should be a skyscraper sheaf $\mathbb{C}_{x}$ for some $x \in C$. Hence for some $\lambda \in \mathbb{C}$, the map given by the composition

$$
V_{1} \stackrel{(i d, \lambda \cdot i d)}{\longrightarrow} V_{1} \oplus V_{1} \stackrel{\alpha \oplus \beta}{\longrightarrow} V
$$

has rank $r_{1}-1$ at $x$. Taking saturation, we get a nonzero map $\widetilde{V_{1}} \rightarrow V$, where $\operatorname{deg} \widetilde{V_{1}}=d_{1}+1$. This contradicts to the stability of $V$ (The final step of this argument appeared in the proof of [6] Lemma 5.6, which was concerned with the case of rank 2).

Definition 2.6. For each integer $\varepsilon$ with $0<\varepsilon<r$, let $R_{\varepsilon}$ be the subset of $\mathcal{M}=S U(r, d)$ consisting of the bundles which admit a subbundle of rank $r_{1}$ and degree $d_{1}$ with $r_{1} d-r d_{1}=\varepsilon$.

Note that $V \in \mathcal{M}$ is not $(1,1)$-stable if and only if $V \in R_{\varepsilon}$ for some $\varepsilon$.

Lemma 2.7. For each $0<\varepsilon<r, R_{\varepsilon}$ is nonempty and irreducible. A general $V \in R_{\varepsilon}$ is contained in the image of $P\left(V_{1}, V_{2}\right)$ for some general $\left(V_{1}, V_{2}\right) \in \mathcal{D}_{\varepsilon}$.

Proof. The nonemptyness of $R_{\varepsilon}$ follows from Lemma 2.4. The remaining statements follow from a more general statement proven by Teixidor i Bigas (see [8], Proposition 1.6). In fact, the case of $U(r, d)$ was worked out there, but the same argument applies to $S U(r, d)$.

\section{Scrolls}

Now we study the intersection of $R_{i}$ and $R_{j}$ inside $\mathcal{M}$ for $0<i \neq j<r$. 
Lemma 3.1. Consider $\left(V_{1}, V_{2}\right) \in \mathcal{D}_{i}$, where $V_{1}$ and $V_{2}$ are Lange-stable. If $i+j \neq r$, then the image of $P\left(V_{1}, V_{2}\right)$ inside $\mathcal{M}$ does not intersect $R_{j}$.

Proof. Consider $V \in P\left(V_{1}, V_{2}\right)$. By Lemma 2.4, there are at most two subbundles, $V_{1}$ and $W_{1}$, which violate the inequality for the $(1,1)$-stability of $V$. Also, $\operatorname{rk}\left(W_{1}\right)=r-\operatorname{rk}\left(V_{1}\right)$ and $\operatorname{deg}\left(W_{1}\right)=d-\operatorname{deg}\left(V_{1}\right)-1$. If $V \in R_{j}$, this means that $V$ admits a subbundle $W_{1}$ such that $j=\operatorname{rk}\left(W_{1}\right) d-r \operatorname{deg}\left(W_{1}\right)$. Hence from the identity (2.2) defining $i$, we see that $i+j=r$.

To study the case $i+j=r$ in more detail, we need first to consider a scroll in $P\left(V_{1}, V_{2}\right)$. For $\left(V_{1}, V_{2}\right) \in \mathcal{D}_{\varepsilon}, 0<\varepsilon<r$, consider the rational map

$$
\Upsilon: \mathbb{P} \operatorname{Hom}\left(V_{2}, V_{1}\right) \rightarrow P\left(V_{1}, V_{2}\right)
$$

defined by the complete linear system

$$
\begin{aligned}
P\left(V_{1}, V_{2}\right) & \cong \mathbb{P} H^{0}\left(C, K_{C} \otimes V_{1}^{*} \otimes V_{2}\right)^{\vee} \\
& \cong \mathbb{P} H^{0}\left(\mathbb{P H o m}\left(V_{2}, V_{1}\right), p^{*} K_{C} \otimes \mathcal{O}(1)\right)^{\vee},
\end{aligned}
$$

where $p: \mathbb{P H o m}\left(V_{2}, V_{1}\right) \rightarrow C$ is the projection.

Lemma 3.2. Assume $g \geq 4$. If $\left(V_{1}, V_{2}\right) \in \mathcal{D}_{\varepsilon}$ is general. Then the map $\Upsilon$ is an embedding.

Proof. For any $x \in C$, the line bundle $p^{*} K_{C} \otimes \mathcal{O}(1)$ restricted to the fiber $p^{-1}(x)$ is very ample. Thus it is very ample on $\operatorname{PHom}\left(V_{2}, V_{1}\right)$ if for every $x, y \in C$, the case $x=y$ included, the restriction map

$$
H^{0}\left(\mathbb{P H o m}\left(V_{2}, V_{1}\right), p^{*} K_{C} \otimes \mathcal{O}(1)\right) \rightarrow H^{0}\left(\pi^{-1}(x+y),\left.p^{*} K_{C} \otimes \mathcal{O}(1)\right|_{\pi^{-1}(x+y)}\right)
$$

is surjective. By the projection formula, this is guaranteed if for any $x, y \in C$,

$$
h^{0}\left(C, K_{C} \otimes V_{1}^{*} \otimes V_{2}\right)-h^{0}\left(C, K_{C}(-x-y) \otimes V_{1}^{*} \otimes V_{2}\right)=2 r_{1} r_{2} .
$$

Since $V_{1}^{*} \otimes V_{2}$ is semistable, this is equivalent to the vanishing of $h^{0}\left(C, V_{2}^{*} \otimes\right.$ $\left.V_{1}(x+y)\right)$. In the below, we will show this vanishing.

If $r=2$, then $V_{1}$ and $V_{2}$ are line bundles and $\varepsilon=1$. In this case, $h^{0}\left(C, V_{2}^{*} \otimes\right.$ $\left.V_{1}(x+y)\right)>0$ for some $x, y$ if and only if $V_{1}^{*} \otimes V_{2} \in C+C-C$ in $\operatorname{Pic}^{1}(C)$. Since $g \geq 4$, we can take $V_{1}^{*} \otimes V_{2}$ outside the locus $C+C-C$.

If $r \geq 3$, we may use the "twisted Brill-Noether for one section" proven by Russo and Teixidor i Bigas([8], Theorem 0.3):

For a general $G \in U\left(r_{G}, d_{G}\right)$, the locus of the bundles $H$ inside $U\left(r_{H}, d_{H}\right)$ such that $h^{0}\left(H^{*} \otimes G\right)>0$ has dimension

$$
\gamma:=r_{H}\left(r_{H}-r_{G}\right)(g-1)+r_{H} d_{G}-r_{G} d_{H},
$$

if it is nonempty.

First assume that $r_{2}>1$ and apply this to $G=V_{1}(x+y)$ and $H=V_{2}$ : for a fixed $G \in U\left(r_{1}, d_{1}+2 r_{1}\right)$, consider the locus of the bundles $H \in S U\left(r_{2}, d_{2}\right)$ admitting a nonzero map $H \rightarrow G$. Since we may choose $\operatorname{det} H$ general by varying $\operatorname{det} G$, we can reduce the above dimension $\gamma$ to $\gamma-g$. We get

$$
\gamma-g=r_{2}\left(r_{2}-r_{1}\right)(g-1)+r_{2}\left(d_{1}+2 r_{1}\right)-r_{1} d_{2}-g
$$


and it can be checked that

$$
=r_{2}^{2}(g-1)-r_{1} r_{2}(g-3)-\varepsilon-g
$$

$$
\gamma-g<\left(r_{2}^{2}-1\right)(g-1)-2=\operatorname{dim} S U\left(r_{2}, d_{2}\right)-2 .
$$

This implies that for a general choice of $\left(V_{1}, V_{2}\right) \in \mathcal{D}_{\varepsilon}$, we get the vanishing of $h^{0}\left(C, V_{2}^{*} \otimes V_{1}(x+y)\right)$ for any $x, y \in C$.

If $r_{2}=1$, then $r_{1}>1$ and the same argument works through for $G=$ $V_{2}^{*}(x+y)$ and $H=V_{1}^{*}$.

Hence for a general $\left(V_{1}, V_{2}\right) \in \mathcal{D}_{\varepsilon}$, we have a scroll $\Upsilon\left(\mathbb{P H o m}\left(V_{2}, V_{1}\right)\right)$ inside $P\left(V_{1}, V_{2}\right)$. This locus provides a criteria on the existence of a subbundle $V^{\prime}$ of rank $r_{2}$ in Lemma 2.4.

Lemma 3.3. Assume $g \geq 4$. Suppose that $V_{1}$ and $V_{2}$ in the nontrivial extension (2.1) are Lange-stable. Then $V$ has a subbundle $V^{\prime} \neq V_{1}$ of rank $r_{2}$ and degree $d_{2}-1$ if and only if the associated point $v \in P\left(V_{1}, V_{2}\right)$ lies on the scroll $\Upsilon\left(\mathbb{P H o m}\left(V_{2}, V_{1}\right)\right)$.

Proof. By Lemma 2.4, the subbundle $V^{\prime} \neq V_{1}$ of rank $r_{2}$ and degree $d_{2}-1$ induces a sheaf injection $V^{\prime}(\rightarrow V) \rightarrow V_{2}$. In other words, there is an elementary transformation

$$
0 \rightarrow V^{\prime} \rightarrow V_{2} \rightarrow \mathbb{C}_{x} \rightarrow 0
$$

for some $x \in C$ which lifts to $V \in H^{1}\left(C, V_{2}^{*} \otimes V_{1}\right)$. This is equivalent to that

$$
v \in \mathbb{P} \operatorname{ker}\left[H^{1}\left(C, V_{2}^{*} \otimes V_{1}\right) \rightarrow H^{1}\left(C,\left(V^{\prime}\right)^{*} \otimes V_{1}\right)\right]
$$

for the induced map on the extension spaces. By definition of $\Upsilon$, this is equivalent to that $v$ lies on the image of the fiber $\left.\mathbb{P H o m}\left(V_{2}, V_{1}\right)\right|_{x}$.

Summarizing the results in this section, we get the following.

Proposition 3.4. For a general $\left(V_{1}, V_{2}\right) \in \mathcal{D}_{i}$ where $0<i<r$, let $P$ be the image of $P\left(V_{1}, V_{2}\right)$ inside $\mathcal{M}$. Let $0<j \neq i<r$.

(1) If $i+j \neq r$, then $P \cap R_{j}$ is empty.

(2) If $i+j=r$, then $P \cap R_{j}$ is the image of the scroll

$$
\Upsilon\left(\mathbb{P H o m}\left(V_{2}, V_{1}\right)\right) \subset P\left(V_{1}, V_{2}\right) .
$$

(3) In particular, $R_{i}$ is not contained in $R_{j}$.

\section{Rational curves}

First we recall Sun's results in [9] on rational curves on $\mathcal{M}$.

Let $\phi: \mathbb{P}^{1} \rightarrow \mathcal{M}$ be a rational curve. Let $X=C \times \mathbb{P}^{1}$ together with the projections $f: X \rightarrow C$ and $\pi: X \rightarrow \mathbb{P}^{1}$. Since $\mathcal{M}$ admits a universal bundle, there is a bundle $E$ on $X$ such that $\phi(t)=\left.E\right|_{C \times\{t\}}$ for each $t \in \mathbb{P}^{1}$. Its restriction to a general fiber $f^{-1}(\xi)=X_{\xi} \cong \mathbb{P}^{1}$ is of the form

$$
\left.E\right|_{X_{\xi}} \cong \bigoplus_{i=1}^{n} \mathcal{O}_{X_{\xi}}\left(\alpha_{1}\right)^{\oplus r_{i}}, \quad \alpha_{1}>\cdots>\alpha_{n} .
$$


Tensoring $E$ by $\pi^{*} \mathcal{O}\left(-\alpha_{n}\right)$, we may assume that $\alpha_{n}=0$ in this generic splitting type of $E$. Then $E$ admits a Harder-Narasimhan filtration

$$
0=E_{0} \subset E_{1} \subset \cdots \subset E_{n}=E
$$

such that the $F_{i}=E_{i} / E_{i-1}$ are torsion free with generic splitting type $\left(\mathcal{O}\left(\alpha_{i}\right)^{\oplus r_{i}}\right)$ for each $i$. Let $F_{i}^{\prime}=F_{i} \otimes \pi^{*} \mathcal{O}_{\mathbb{P}^{1}}\left(-\alpha_{i}\right)$ so that they have generic splitting type $\left(\mathcal{O}^{\oplus r_{i}}\right)$. Let $\operatorname{deg}\left(E_{i}\right)$ denote the degree of $E_{i}$ on the generic fiber of $\pi$.

Lemma 4.1 ([9], (2.1), (2.2), Lemma 2.2). (1) For the ample generator $\mathcal{L}_{\mathcal{M}}$ of $\operatorname{Pic}(\mathcal{M})$, we have

$\operatorname{deg} \phi^{*}\left(\mathcal{L}_{\mathcal{M}}\right)=r \sum_{i=1}^{n} c_{2}\left(F_{i}^{\prime}\right)+\sum_{i=1}^{n-1}\left[r k\left(E_{i}\right) \operatorname{deg}(E)-r k(E) \operatorname{deg}\left(E_{i}\right)\right]\left(\alpha_{i}-\alpha_{i+1}\right)$.

(2) Any torsion free sheaf $\mathcal{E}$ on $X$ with generic splitting type $\left(\mathcal{O}^{\oplus r_{i}}\right)$ must have $c_{2}(\mathcal{E}) \geq 0$. Also, $c_{2}(\mathcal{E})=0$ if and only if $\mathcal{E}=f^{*} W$ where $W$ is a locally free sheaf on $C$.

Combining the lemma with our discussion in previous sections, we get the following.

Proposition 4.2. Assume $g \geq 4$, let $0<\varepsilon<r$.

(1) For a general $\left(V_{1}, V_{2}\right) \in \mathcal{D}_{\varepsilon}$, consider the morphism $P\left(V_{1}, V_{2}\right) \rightarrow \mathcal{M}$. For every line on $P\left(V_{1}, V_{2}\right)$, its image inside $\mathcal{M}$ has degree $\varepsilon$.

(2) Any smooth rational curve $\phi$ on $\mathcal{M}$ of degree $\varepsilon$ which passes through a general point of $R_{\varepsilon}$ is an image of a line on $P\left(V_{1}, V_{2}\right)$ for some $\left(V_{1}, V_{2}\right) \in \mathcal{D}_{\varepsilon}$.

(3) For each $\varepsilon$, the locus swept out by the rational curves on $\mathcal{M}$ of degree $\leq \varepsilon$ consists of $\varepsilon$ irreducible components whose closures are given by $R_{1}, \ldots, R_{\varepsilon}$.

Proof. (1) This is immediate from (4.1).

(2) By Lemma 2.7, a general point $V$ of $R_{\varepsilon}$ is contained in the image of $P\left(V_{1}, V_{2}\right)$ for some general $\left(V_{1}, V_{2}\right) \in \mathcal{D}_{\varepsilon}$. By Lemma 2.4 and Lemma 3.3, $V_{1}$ is the unique subbundle of $V$ violating the inequality for the $(1,1)$-stability of $V$.

Now assume that $\phi$ passes through $V \in R_{\varepsilon}$. By (4.1), $\operatorname{deg} \phi=\varepsilon<r$ implies that $n=2, c_{2}\left(F_{i}^{\prime}\right)=0$, and $\alpha_{1}=1$. Also by Lemma $4.1(2)$,

$$
F_{1}=E_{1}=f^{*}\left(V_{1}\right) \otimes \pi^{*} \mathcal{O}_{\mathbb{P}^{1}}(1) \text { and } F_{2}=E_{2} / E_{1}=f^{*}\left(V_{2}\right) .
$$

Hence we get the exact sequence

$$
0 \rightarrow f^{*}\left(V_{1}\right) \otimes \pi^{*} \mathcal{O}_{\mathbb{P}^{1}}(1) \rightarrow E \rightarrow f^{*}\left(V_{2}\right) \rightarrow 0 .
$$

This shows that $\phi$ is an image of a line on $P\left(V_{1}, V_{2}\right)$.

(3) By Proposition 3.4(3), $R_{i}$ is not contained in $R_{j}$ if $j \neq i$. The images of $P\left(V_{1}, V_{2}\right)$ for $\left(V_{1}, V_{2}\right) \in \mathcal{D}_{\varepsilon}$ are dense in $R_{\varepsilon}$ by Lemma 2.7. By Proposition $4.2(1)$, the images of the lines on $P\left(V_{1}, V_{2}\right)$ are rational curves of degree $\varepsilon$. Hence it suffices to show that any rational curve $\phi$ of degree $\varepsilon$ is contained in $R_{1} \cup \cdots \cup R_{\varepsilon}$. Indeed, by the formula (4.1), every point on a curve $\phi$ of 
degree $\varepsilon<r$ corresponds to a bundle admitting a subbundle $V_{1}$ such that $\operatorname{rk}\left(V_{1}\right) d-r \operatorname{deg}\left(V_{1}\right) \leq \varepsilon$.

For $\varepsilon=1$, it is easy to see that there are a projective bundle $\mathcal{P} \rightarrow \mathcal{D}_{1}$ whose fiber at $\left(V_{1}, V_{2}\right)$ is $P\left(V_{1}, V_{2}\right)$, and a universal bundle $U \rightarrow C \times \mathcal{P}$ which induces a rational map $\mathcal{P} \rightarrow \mathcal{M}$. In this case, a stronger version of Proposition 4.2(2) has been proven.

Proposition 4.3. (1) ([9], Theorem 2) The classifying map $\mathcal{P} \rightarrow \mathcal{M}$ is a morphism whose image coincides with $R_{1}$. And every line on $\mathcal{M}$ is an image of a line on $P\left(V_{1}, V_{2}\right)$ for some $\left(V_{1}, V_{2}\right) \in \mathcal{D}_{1}$.

(2) ([5], §4) Moreover, $\Phi: P\left(V_{1}, V_{2}\right) \rightarrow \mathcal{M}$ is an embedding for a general $\left(V_{1}, V_{2}\right) \in \mathcal{D}_{1}$.

Consider a general $\left(V_{1}, V_{2}\right) \in \mathcal{D}_{1}$ so that $P\left(V_{1}, V_{2}\right)$ is embedded in $\mathcal{M}$. We denote the image by $P$. By formula $(4.1),\left.\mathcal{L}_{\mathcal{M}}\right|_{P} \cong \mathcal{O}_{P}(1)$. The converse also holds.

Lemma 4.4. Assume $g \geq 4$. Let $P$ be a subvariety of $\mathcal{M}$ passing through a general point of $R_{1}$ such that $P \cong \mathbb{P}^{k}$ for some $k$ and $\left.\mathcal{L}_{\mathcal{M}}\right|_{P} \cong \mathcal{O}_{P}(1)$. Then $P$ is the image of a $k$-dimensional linear subspace of $P\left(V_{1}, V_{2}\right)$ for some $\left(V_{1}, V_{2}\right) \in \mathcal{D}_{1}$.

Proof. As before, we may assume that $P$ passes through an image of the point $V \in P\left(V_{1}, V_{2}\right)$ for a general $\left(V_{1}, V_{2}\right) \in \mathcal{D}_{1}$, such that $V_{1}$ is the unique subbundle violating the (1,1)-stability of $V$. The proof of Proposition 4.2(2) shows that every line on $P$ passing through $V$ is an image of a line on $P\left(V_{1}, V_{2}\right)$. Hence $P$ is contained in the image of $P\left(V_{1}, V_{2}\right)$.

Finally we give another proof of nonabelian Torelli theorem based on the above discussions. As was mentioned in $\S 1$, Sun [9] found a way to recover the curve $C$ from the geometry of rational curves on $\mathcal{M}=S U(r, d)$ of degree $r$ passing through a general point of $\mathcal{M}$. In the following, we give a way to recover $C$ from the geometry of rational curves on $\mathcal{M}$ of degree 1 and $r-1$, which sweep out a small sublocus of $\mathcal{M}$.

Proposition 4.5. Let $C_{1}$ and $C_{2}$ be smooth algebraic curves of genus $g \geq 4$. If $S U_{C_{1}}(r, d) \cong S U_{C_{2}}(r, d)$, then $C_{1} \cong C_{2}$.

Proof. By Proposition 4.2(3), each irreducible variety $R_{\varepsilon}, 0<\varepsilon<r$, is characterized in terms of the rational curves of degree $\leq \varepsilon$. More precisely, $R_{\varepsilon}$ is the closure of $R_{\varepsilon}^{\circ} \backslash R_{\varepsilon-1}^{\circ}$, where $R_{k}^{\circ}$ is the sublocus swept out by the rational curves of degree $\leq k$.

By Lemma 4.4, we can pick out a subvariety $P$ of $R_{1}$ which is the image of $P\left(V_{1}, V_{2}\right)$ for a general $\left(V_{1}, V_{2}\right) \in \mathcal{D}_{1}$. Note that the composition $\mathbb{P H o m}\left(V_{2}, V_{1}\right) \rightarrow P\left(V_{1}, V_{2}\right) \rightarrow \mathcal{M}$ is an embedding by Lemma 3.2 and Proposition 4.3(2). If $r>2$, then the locus $P \cap R_{r-1}$ is isomorphic to a scroll over $C$ by Proposition $3.4(2)$. 
Now assume $r=2$. We claim that the locus of points $v \in P$ such that $v \in l$ for a line $l$ on $\mathcal{M}$ which is not contained in $P$, is isomorphic to $C$. Indeed, if there is a line $l$ on $\mathcal{M}$ which is not contained in $P$ such that $v \in l \cap P$, then the associated bundle $V$ admits two different (line) subbundles $V_{1}$ and $V_{1}^{\prime}$ of degree $d_{1}\left(=d_{2}-1\right)$ by Proposition $4.3(1)$, and vice versa. By Lemma 3.3, the locus of such bundles inside $P$ is the image of $\operatorname{PHom}\left(V_{2}, V_{1}\right) \cong C$.

In this way, we can recover $C$ from $\mathcal{M}=S U_{C}(r, d)$ using the properties of $\mathcal{M}$ as a projective variety with $\operatorname{Pic}(\mathcal{M}) \cong \mathbb{Z}$.

Acknowledgement. The author would like to thank Professor Jun-Muk Hwang for the suggestions on the earlier version of this paper, and the referee for several suggestions which were useful to improve the presentation.

\section{References}

[1] J.-M. Drezet and M. S. Narasimhan, Groupe de Picard des variétés de modules de fibrés semi-stables sur les courbes algébriques, Invent. Math. 97 (1989), no. 1, 53-94.

[2] J.-M. Hwang, Hecke curves on the moduli space of vector bundles over an algebraic curve, Proceedings of the Symposium Algebraic Geometry in East Asia, Kyoto. (2001), 155-164.

[3] J. Kollár, Rational Curves on Algebraic Varieties, Springer-Verlag, Berlin, 1996.

[4] H. Lange, Zur Klassifikation von Regelmannigfaltigkeiten, Math. Ann. 262 (1983), no. 4, 447-459.

[5] N. Mok and X. Sun, Remarks on lines and minimal rational curves, Sciences in China Series A: Mathematics 52 (2009), no. 6, 1-16.

[6] M. S. Narasimhan and S. Ramanan, Geometry of Hecke cycles. I, C. P. Ramanujam-a tribute, pp. 291-345, Tata Inst. Fund. Res. Studies in Math., 8, Springer, Berlin-New York, 1978 .

[7] S. Ramanan, The moduli spaces of vector bundles over an algebraic curve, Math. Ann. 200 (1973), 69-84.

[8] B. Russo and M. Teixidor i Bigas, On a conjecture of Lange, J. Algebraic Geom. 8 (1999), no. 3, 483-496

[9] X. Sun, Minimal rational curves on moduli spaces of stable bundles, Math. Ann. 331 (2005), no. 4, 925-937.

Department of Mathematics

KonKUK UNIVERSITY

SEOUL 143-702, KOREA

E-mail address: ischoe@konkuk.ac.kr 